\title{
Molecular Dynamics Simulations of the Adsorption of Phthalate Esters on Smectite Clay Surfaces
}

\author{
Jennifer A.R. Willemsen*, Satish C.B. Myneni^, Ian C. Bourg* \\ *Department of Civil \& Environmental Engineering, Princeton University, Princeton, NJ 08544, United States \\ ${ }^{\wedge}$ Department of Geoscience, Princeton University, Princeton, NJ 08544, United States
}

Supplementary Information 


\section{DMP Literature Data}

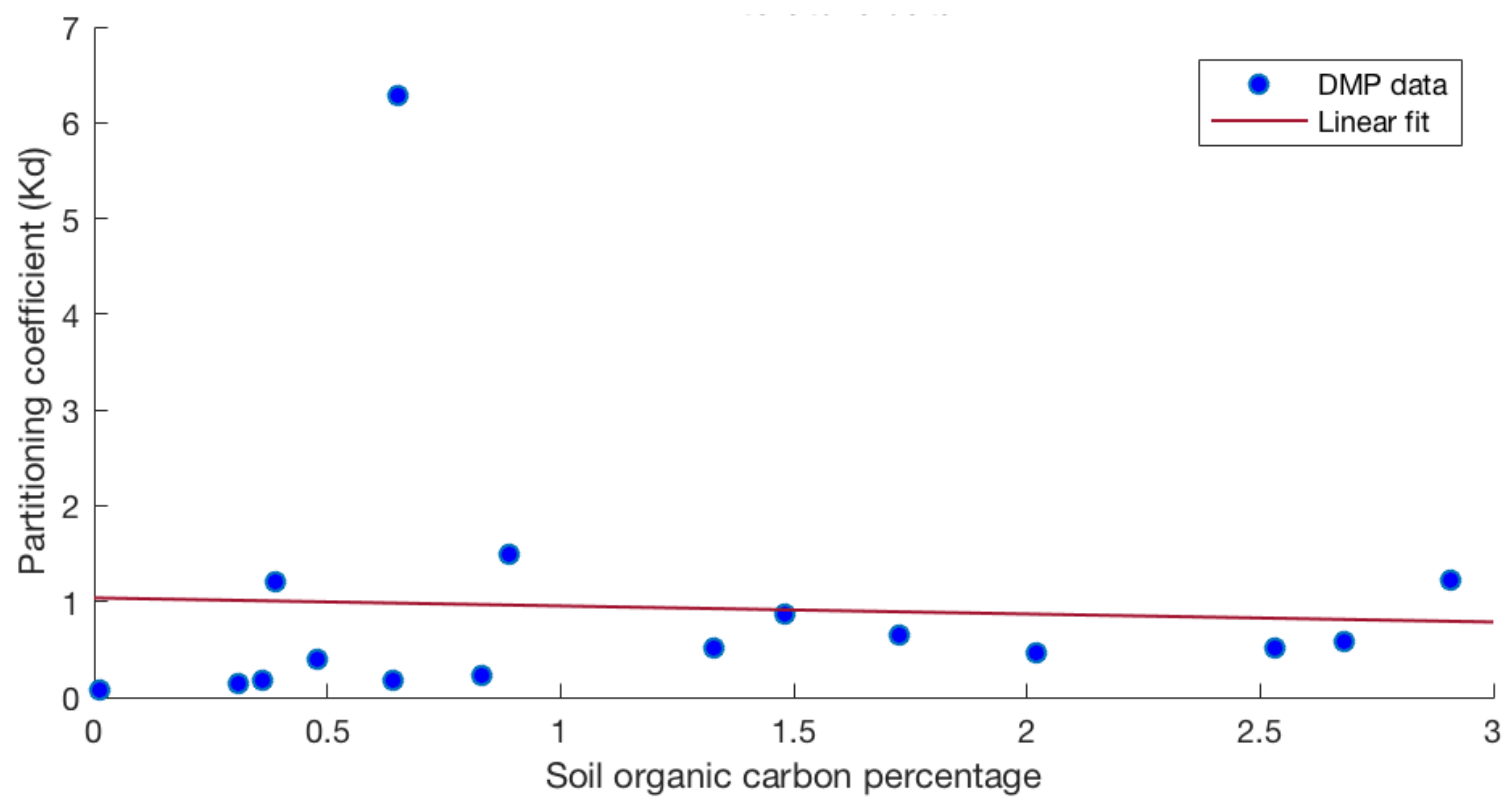

Figure S1: Compilation of dimethyl phthalate (DMP) $K_{d}$ values vs. soil organic carbon percentage $\left(f_{\text {oc }}\right)$ reported in previous studies of DMP adsorption in soils. ${ }^{1-7}$ The data points span a range of salinities and $\mathrm{pH}$ values. 


\section{Molecular Dynamics Simulation Parameters}

Clay edge site partial charges were calculated using an approach developed by Lammers et al. $(2017)^{8}$ which takes into account bond valence, charge saturation, and cation charge to calculate CLAYFF-compatible partial charges for edge $\mathrm{O}$ atoms using the equation:

$Z_{O e j}^{P}=-2.00+\sum_{i} \frac{N_{i}\left(Z_{i}-Z_{i}^{P}\right)}{C N_{i}}$

where $\mathrm{N}_{\mathrm{i}}$ is the number of cations coordinated to the edge oxygen, $\mathrm{Z}_{\mathrm{i}}$ is the cation valence, $\mathrm{Z}_{\mathrm{i}}^{\mathrm{P}}$ is the cation partial charge, and $\mathrm{CN}_{\mathrm{i}}$ is the number of $\mathrm{O}$ atoms coordinated to the cation.

Table S1: Interatomic potentials for clay minerals ${ }^{8-9}$, water ${ }^{10}$, cations ${ }^{11-12}$, and phthalate esters ${ }^{13}$. The last two columns define the Lennard-Jones 6-12 model of the Van der Waals interaction potential between identical atoms: $\phi_{\mathrm{LJ}}=4 \varepsilon \times\left[(\sigma / r)^{12}-(\sigma / r)^{6}\right]$, where $\phi$ is the interaction potential and $r$ is the interatomic distance.

\begin{tabular}{|c|c|c|c|}
\hline Atom & Partial Charge (e) & $\varepsilon(\mathrm{eV})$ & $\sigma(\AA)$ \\
\hline \multicolumn{4}{|c|}{ Clay Mineral Parameters } \\
\hline $\mathbf{S i} \mathbf{i}^{\mathrm{Tet}}$ & 2.10000 & $7.98102 \mathrm{e}-8$ & 3.30203 \\
\hline $\mathbf{A l}^{\text {Oct }}$ & 1.57500 & $5.76646 \mathrm{e}-8$ & 4.27124 \\
\hline $\mathbf{M g}^{\text {Oct }}$ & 1.35998 & $3.91562 \mathrm{e}-8$ & 5.26432 \\
\hline Bridging $\mathbf{O}$ & -1.05000 & $6.73853 \mathrm{e}-3$ & 3.16556 \\
\hline$>\mathrm{Si}-\mathrm{OH}$ & -0.95000 & $6.73853 \mathrm{e}-3$ & 3.16556 \\
\hline$>\mathrm{Si}-\mathrm{OH}$ & 0.42500 & 0.0 & 0.0 \\
\hline$>\mathrm{Mg}^{\mathrm{Oct}}-\mathrm{OH}-\mathrm{Al}^{\mathrm{Oct}}<$ & -1.08083 & $6.73853 \mathrm{e}-3$ & 3.16556 \\
\hline$>\mathrm{Mg}^{\mathrm{Oct}}-\mathbf{O}-\mathrm{Si}^{\mathrm{Tet}}<$ & -1.18083 & $6.73853 e-3$ & 3.16556 \\
\hline Edge $>\mathrm{Si}-\mathrm{OH}$ & -0.95000 & $6.73853 e-3$ & 3.16556 \\
\hline$E d g e>\mathrm{Al}^{\mathrm{Oct}}-\mathrm{OH}-\mathrm{Al}^{\mathrm{Oct}}<$ & -0.95000 & $6.73853 e-3$ & 3.16556 \\
\hline$E d g e>\mathrm{Al}^{\mathrm{Oct}}-\mathbf{O}-\mathrm{Si}^{\mathrm{Tet}}<$ & -1.28750 & $6.73853 e-3$ & 3.16556 \\
\hline$E d g e>\mathrm{Mg}^{\mathrm{Oct}}-\mathrm{OH}-\mathrm{Al}^{\mathrm{Oct}}<$ & -1.08083 & $6.73853 e-3$ & 3.16556 \\
\hline$E d g e>\mathrm{Al}-\mathrm{OH}_{2}$ & -0.84760 & $6.73853 \mathrm{e}-3$ & 3.16556 \\
\hline$E d g e>\mathrm{Al}-\mathrm{OH}_{2}$ & 0.42380 & 0.0 & 0.0 \\
\hline$E d g e>\mathrm{Mg}^{\mathrm{Oct}}-\mathbf{O}-\mathrm{Si}^{\mathrm{Tet}}<$ & -1.41833 & $6.73853 e-3$ & 3.16556 \\
\hline Edge $>\mathrm{Mg}-\mathrm{OH}_{2}$ & -0.84760 & $6.73853 e-3$ & 3.16556 \\
\hline Edge $\mathbf{A l}^{3+}$ & 1.81250 & $5.76646 \mathrm{e}-8$ & 4.27124 \\
\hline Edge $\mathbf{M g}^{2+}$ & 1.46665 & $3.91562 \mathrm{e}-8$ & 5.26432 \\
\hline
\end{tabular}

Water Molecule Parameters

$\begin{array}{ccccc}\mathbf{O} & -0.84760 & 6.73853 \mathrm{e}-3 & 3.16556 & 0.0 \\ \mathbf{H} & 0.42380 & 0.0 & \\ & \text { Cation Parameters } & & 2.36084 \\ \mathbf{C a}^{2+} & 2.00000 & 1.95028 \mathrm{e}-2 & \end{array}$




\begin{tabular}{|c|c|c|c|}
\hline $\mathrm{Cl}^{-}$ & -1.00000 & $4.33633 \mathrm{e}-3$ & 4.40000 \\
\hline \multicolumn{4}{|c|}{ Phthalate Esters } \\
\hline Aromatic $\mathbf{C}$ & -0.11500 & $3.0354 \mathrm{e}-3$ & 3.55 \\
\hline Aromatic $\mathbf{H}$ & 0.11500 & $1.3009 \mathrm{e}-3$ & 2.42 \\
\hline Ester $\mathrm{C}^{\text {Aro }}-\mathbf{C}-\mathrm{OO}$ & 0.62500 & $4.5531 \mathrm{e}-3$ & 3.75 \\
\hline Ester $\mathbf{O}=\mathrm{C}$ & -0.43000 & $9.1063 \mathrm{e}-3$ & 2.96 \\
\hline Ester $\mathrm{OC}-\mathbf{O}-\mathrm{R}$ & -0.33000 & $7.3718 \mathrm{e}-3$ & 3.00 \\
\hline Ester $\mathrm{O}-\mathbf{C H}_{2}-\mathrm{R}$ & 0.19000 & $2.8620 \mathrm{e}-3$ & 3.50 \\
\hline Ester $\mathrm{O}-\mathrm{CH}_{2}-\mathrm{R}$ & 0.03000 & $6.5045 \mathrm{e}-4$ & 2.42 \\
\hline Alkane $\mathrm{R}_{2}-\mathbf{C H}_{2}$ & -0.12000 & $2.8620 \mathrm{e}-3$ & 3.50 \\
\hline Alkane $\mathrm{R}_{2}-\mathrm{CH}_{2}$ & 0.06000 & $1.3009 \mathrm{e}-3$ & 2.50 \\
\hline Alkane $\mathrm{R}-\mathrm{CH}_{3}$ & -0.18000 & $2.8620 \mathrm{e}-3$ & 3.50 \\
\hline Alkane $\mathrm{R}-\mathrm{CH}_{3}$ & 0.06000 & $1.3009 \mathrm{e}-3$ & 2.50 \\
\hline BP Ester $\mathrm{O}-\mathrm{CH}_{2}-\mathrm{C}^{\text {Aro }}$ & 0.30500 & $2.8620 \mathrm{e}-3$ & 3.50 \\
\hline
\end{tabular}

*Parameters for this specific atom in benzyl butyl phthalate (BBP) could not be identified in OPLS-AA. We assigned this atom the same LJ parameters as for OPLS-AA ester carbons and a partial charge such that the overall BBP molecule charge was neutral. In visual inspection of our simulation trajectories, the resulting BBP molecules exhibited no unexpected behavior.

Of note: The SPC/E and CLAYFF force fields use different combining rules and a different convention for Lennard-Jones and Coulombic interactions between bonded atoms (LAMMPS "special bonds" command) than the OPLS-AA force field. For our simulations, we always followed the conventions of the SPC/E and CLAYFF force fields since these accounted for the majority of atoms in our simulations. 


\section{Batch Adsorption Experiments}

Procedure: DMP solutions were prepared at concentrations ranging from 5 to $80 \mu \mathrm{M}$ in $0.1 \mathrm{M} \mathrm{CaCl}_{2}$ from and DEP solutions were prepared at concentrations ranging from 0.5 to $20 \mu \mathrm{M}$ in $0.1 \mathrm{M} \mathrm{CaCl}_{2}$ (pH = 5.30). Experiments were carried out in 50 $\mathrm{mL}$ glass centrifuge tubes containing $20 \mathrm{mg}$ of purified clay and $20 \mathrm{~mL}$ of solution. Each experiment was run in triplicate, and control samples were run with either no clay or no phthalate. Each tube was wrapped in foil to prevent photodegradation and placed on a bench-top shaker $(160 \mathrm{rpm})$ at room temperature for five days as required to reach steady state. ${ }^{14}$ Finally, the tubes were centrifuged for 30 minutes at $4500 \mathrm{rpm}$ and the supernatant solutions were recovered and filtered using $0.2 \mathrm{micron}$ nylon filters into glass vials. Clay slurries and supernatant solutions were stored at $4{ }^{\circ} \mathrm{C}$. DEP solution sample concentration was analyzed within 1 day by fluorescence spectroscopy with an excitation wavelength of $279 \mathrm{~nm}$ and an emission wavelength of $347 \mathrm{~nm}$. DMP samples were analyzed using UV spectroscopy at a wavelength of $230 \mathrm{~nm}$. Adsorbed concentration $q$ (mol kg$\left.{ }^{1}\right)$ was determined from the aqueous phthalate concentration at steady state $C\left(\mathrm{~mol} \mathrm{dm}^{-3}\right)$, its known starting concentration $C_{\text {initial, }}$, and the ratio of water volume to clay mass $\left(V / m=10^{-3} \mathrm{dm}^{3} \mathrm{~kg}^{-1}\right)$ using the mass balance relation $q=\left(C_{\text {initial }}-C\right) \times(V / m)$. In addition, DBP experiments were conducted at various solid to solution ratios (1 mg: $1 \mathrm{~mL}$ and $1 \mathrm{mg}: 30 \mathrm{~mL})$. Final solution concentrations were too close to the detection limit of our instrument to confidently report a partition coefficient, but the results do suggest that DBP adsorption is at least 100x stronger than DEP.
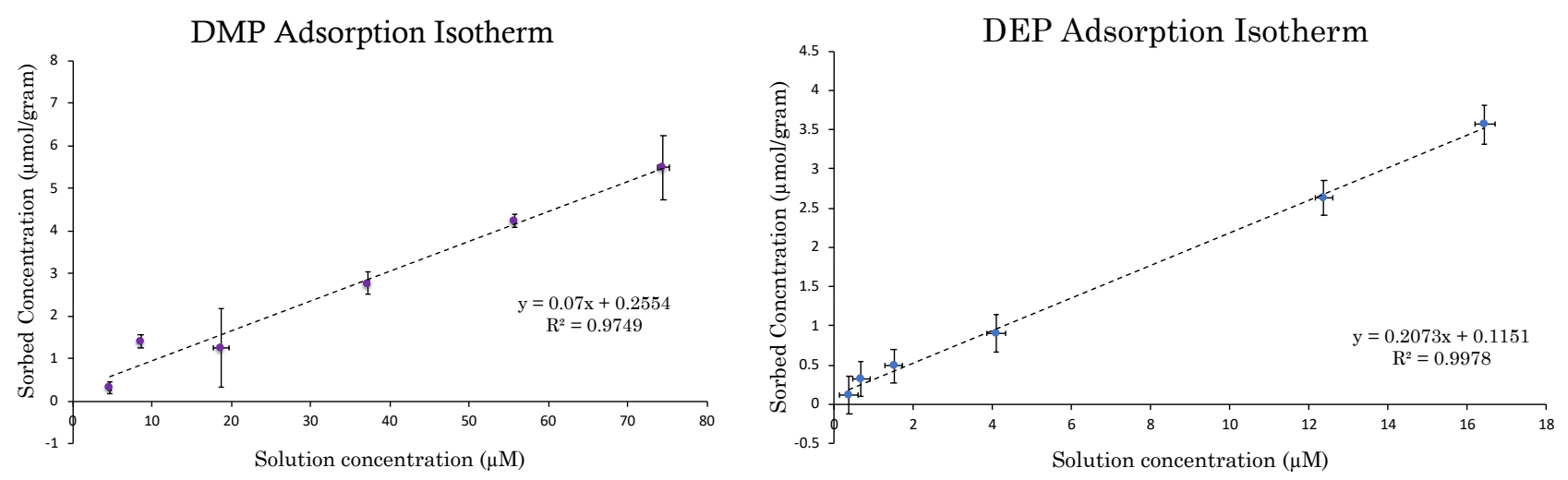

Figure S2: Adsorption isotherms for DMP (left) and DEP (right). Each point is the average of three replicates and error bars represent 95\% CI. All isotherms were linear over this concentration range. 


\section{X-Ray Diffraction Experiments}

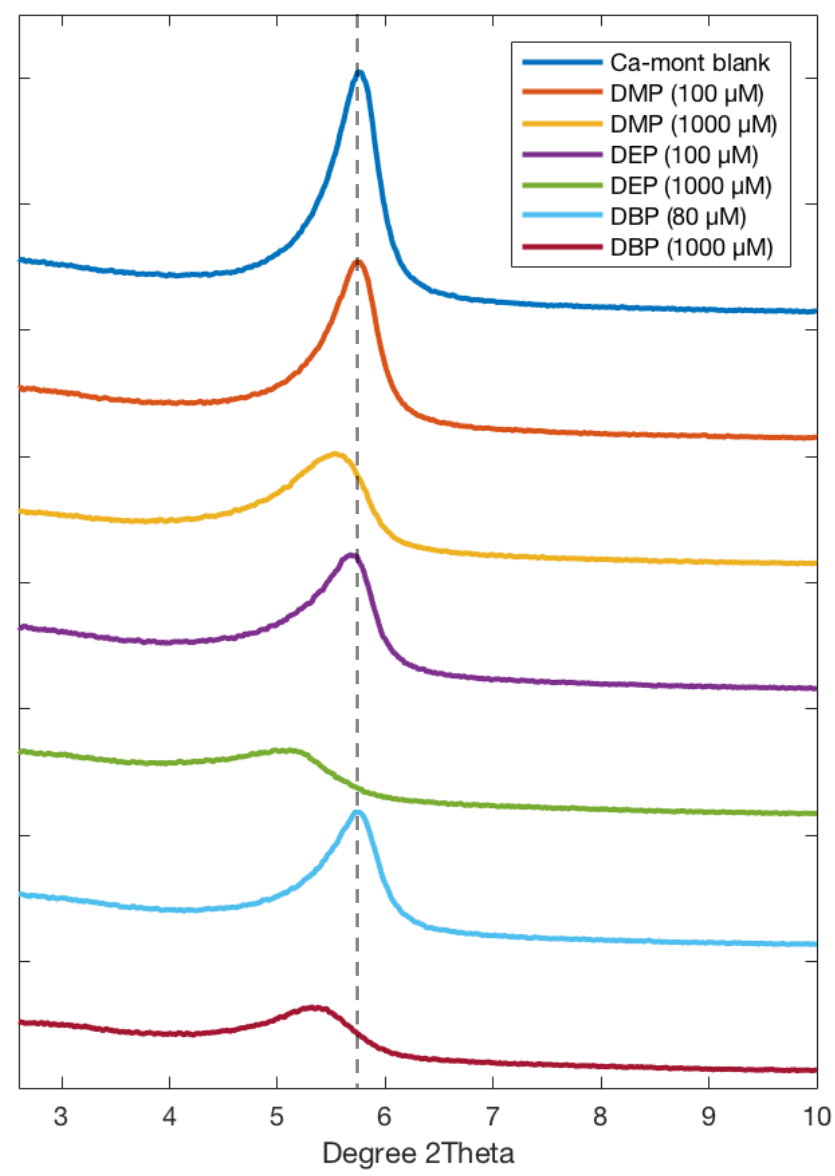

Figure S3: XRD results for clay samples exposed to $\mathrm{CaCl}_{2}$ only (blank), $100 \mu \mathrm{M}$ DMP, $1000 \mu \mathrm{M}$ DMP, $100 \mu \mathrm{M}$ DEP, $1000 \mu \mathrm{M}$ DEP, $80 \mu \mathrm{M}$ DBP, and $1000 \mu \mathrm{M}$ DBP.

Table S2: Peak maxima and corresponding clay basal spacings calculated from the XRD data shown in Figure S3.

\begin{tabular}{|c|c|c|}
\hline Sample & Peak location $(2 \theta)$ & do01 $(\AA)$ \\
\hline Ca-mont blank & 5.76 & 15.4 \\
\hline DMP $(100 \mu \mathrm{M})$ & 5.76 & 15.4 \\
\hline DMP $(1000 \mu \mathrm{M})$ & 5.54 & 16.0 \\
\hline DEP $(100 \mu \mathrm{M})$ & 5.68 & 15.5 \\
\hline DEP $(1000 \mu \mathrm{M})$ & 5.12 & 17.3 \\
\hline DBP $(80 \mu \mathrm{M})$ & 5.72 & 15.4 \\
\hline DBP $(1000 \mu \mathrm{M})$ & 5.32 & 16.6 \\
\hline
\end{tabular}




\section{Simulation Density Profiles}
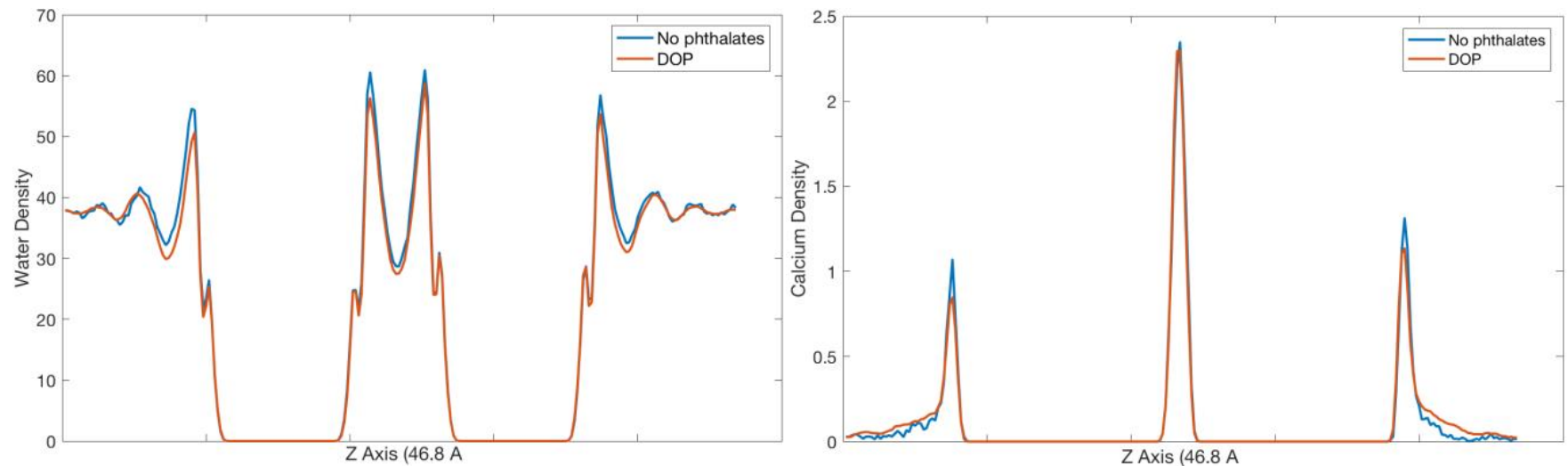

Figure S4: Water (left) and calcium (right) density profiles along the z-axis of the simulation box for a simulation containing no phthalate esters (blue) and the simulation containing three DOP molecules. The number of calcium and water molecules were consistent between simulations. 


\section{Simulation Energetics}

Table S3: Total pairwise interaction potential between the phthalate molecule its surroundings in the interlayer, bottom, top and bulk regions and its breakdown into Coulomb and Van der Waals interactions. All values are in eV and reported errors represent 95\% CI.

\begin{tabular}{|c|c|c|c|c|c|c|c|c|c|c|c|c|}
\hline \multirow{2}{*}{ Phthalate } & \multicolumn{3}{|c|}{ Interlayer } & \multicolumn{3}{c|}{ Bottom } & \multicolumn{3}{c|}{ Top } & \multicolumn{2}{c|}{ Bulk } \\
\cline { 2 - 13 } & Total & VdW & Coul & Total & VdW & Coul & Total & VdW & Coul & Total & VdW & Coul \\
\hline \multirow{2}{*}{ DMP } & -0.79 & -0.49 & -0.30 & -0.78 & -0.48 & -0.30 & -0.79 & -0.46 & -0.30 & -0.75 & -0.42 & -0.32 \\
& \pm 0.01 & \pm 0.01 & \pm 0.01 & \pm 0.01 & \pm 0.01 & \pm 0.01 & \pm 0.02 & \pm 0.01 & \pm 0.01 & \pm 0.01 & \pm 0.01 & \pm 0.01 \\
\hline \multirow{2}{*}{ DEP } & -0.87 & -0.59 & -0.28 & -0.85 & -0.56 & -0.30 & -0.85 & -0.54 & -0.32 & -0.79 & -0.49 & -0.31 \\
& \pm 0.01 & \pm 0.01 & \pm 0.01 & \pm 0.01 & \pm 0.01 & \pm 0.01 & \pm 0.01 & \pm 0.01 & \pm 0.01 & \pm 0.01 & \pm 0.01 & \pm 0.01 \\
\hline \multirow{2}{*}{ DBP } & -0.93 & -0.68 & -0.25 & -0.97 & -0.67 & -0.29 & -1.00 & -0.70 & -0.30 & -0.91 & -0.60 & -0.31 \\
& \pm 0.01 & \pm 0.01 & \pm 0.01 & \pm 0.01 & \pm 0.01 & \pm 0.01 & \pm 0.01 & \pm 0.01 & \pm 0.01 & \pm 0.03 & \pm 0.03 & \pm 0.01 \\
\hline \multirow{2}{*}{ BBP } & -1.23 & -0.83 & -0.39 & -1.12 & -0.77 & -0.35 & -1.16 & -0.76 & -0.39 & -1.07 & -0.69 & -0.38 \\
& \pm 0.07 & \pm 0.04 & \pm 0.03 & \pm 0.01 & \pm 0.01 & \pm 0.01 & \pm 0.02 & \pm 0.01 & \pm 0.01 & \pm 0.02 & \pm 0.01 & \pm 0.02 \\
\hline \multirow{2}{*}{ DEHP } & -1.25 & -0.94 & -0.32 & -1.25 & -0.96 & -0.29 & -1.23 & -0.88 & -0.36 & -1.14 & -0.86 & -0.28 \\
& \pm 0.01 & \pm 0.01 & \pm 0.01 & \pm 0.03 & \pm 0.02 & \pm 0.01 & \pm 0.02 & \pm 0.02 & \pm 0.03 & \pm 0.01 & \pm 0.01 & \pm 0.01 \\
\hline \multirow{2}{*}{ DOP } & -1.20 & -0.92 & -0.28 & -1.28 & -0.97 & -0.31 & -1.28 & -0.97 & -0.32 & -1.20 & -0.93 & -0.26 \\
& \pm 0.01 & \pm 0.01 & \pm 0.01 & \pm 0.01 & \pm 0.01 & \pm 0.01 & \pm 0.01 & \pm 0.01 & \pm 0.01 & \pm 0.02 & \pm 0.02 & \pm 0.01 \\
\hline
\end{tabular}

Table S4: Free energies, enthalpies, and entropies of adsorption for the interlayer, bottom and top regions along with the overall system. All values are in $\mathrm{eV}$ and reported errors represent $95 \% \mathrm{CI}$.

\begin{tabular}{|c|c|c|c|c|c|c|c|c|c|c|c|c|}
\hline \multirow{2}{*}{ Phthalate } & \multicolumn{3}{|c|}{ Interlayer } & \multicolumn{3}{|c|}{ Bottom } & \multicolumn{3}{|c|}{ Top } & \multicolumn{3}{|c|}{ Overall } \\
\hline & $\Delta \mathrm{F}$ & $\Delta \mathrm{H}$ & $-\mathrm{T} \Delta \mathrm{S}$ & $\Delta \mathrm{F}$ & $\Delta \mathrm{H}$ & $-\mathrm{T} \Delta \mathrm{S}$ & $\Delta \mathrm{F}$ & $\Delta \mathrm{H}$ & $-\mathrm{T} \Delta \mathrm{S}$ & $\Delta \mathrm{F}$ & $\Delta \mathrm{H}$ & $-\mathrm{T} \Delta \mathrm{S}$ \\
\hline \multirow{2}{*}{ DMP } & -0.14 & -0.04 & -0.10 & -0.24 & -0.03 & -0.21 & -0.04 & -0.04 & -0.002 & -0.22 & -0.04 & -0.18 \\
\hline & \pm 0.06 & \pm 0.02 & \pm 0.06 & \pm 0.01 & \pm 0.01 & \pm 0.02 & \pm 0.01 & \pm 0.02 & \pm 0.03 & \pm 0.03 & \pm 0.02 & \pm 0.04 \\
\hline \multirow{2}{*}{ DEP } & -0.15 & -0.08 & -0.07 & -0.26 & -0.04 & -0.22 & -0.04 & -0.06 & 0.02 & -0.23 & -0.06 & -0.17 \\
\hline & \pm 0.04 & \pm 0.01 & \pm 0.04 & \pm 0.05 & \pm 0.02 & \pm 0.05 & \pm 0.01 & \pm 0.02 & \pm 0.02 & \pm 0.05 & \pm 0.03 & \pm 0.06 \\
\hline \multirow{2}{*}{ DBP } & -0.12 & -0.02 & -0.10 & -0.40 & -0.06 & -0.34 & 0.01 & -0.09 & 0.10 & -0.37 & -0.06 & -0.31 \\
\hline & \pm 0.02 & \pm 0.03 & \pm 0.04 & \pm 0.05 & \pm 0.03 & \pm 0.06 & \pm 0.05 & \pm 0.03 & \pm 0.06 & \pm 0.05 & \pm 0.05 & \pm 0.07 \\
\hline \multirow{2}{*}{ BBP } & -0.29 & -0.07 & -0.22 & -0.22 & -0.05 & -0.17 & -0.06 & -0.08 & 0.02 & -0.26 & -0.07 & -0.19 \\
\hline & \pm 0.04 & \pm 0.07 & \pm 0.08 & \pm 0.05 & \pm 0.03 & \pm 0.06 & \pm 0.04 & \pm 0.03 & \pm 0.05 & \pm 0.04 & \pm 0.07 & \pm 0.08 \\
\hline \multirow{2}{*}{ DEHP } & 0.19 & -0.12 & 0.31 & -0.44 & -0.09 & -0.35 & 0.15 & -0.08 & 0.23 & -0.42 & -0.10 & -0.32 \\
\hline & \pm 0.23 & \pm 0.02 & \pm 0.23 & \pm 0.13 & \pm 0.03 & \pm 0.13 & \pm 0.05 & \pm 0.02 & \pm 0.05 & \pm 0.13 & \pm 0.03 & \pm 0.13 \\
\hline \multirow{2}{*}{ DOP } & -0.30 & -0.001 & -0.30 & -0.57 & -0.09 & -0.48 & -0.17 & -0.10 & -0.07 & -0.54 & -0.06 & -0.48 \\
\hline & \pm 0.03 & \pm 0.02 & \pm 0.04 & \pm 0.13 & \pm 0.02 & \pm 0.13 & \pm 0.08 & \pm 0.02 & \pm 0.08 & \pm 0.12 & \pm 0.05 & \pm 0.13 \\
\hline
\end{tabular}




\section{References}

(1) Zhao, X.-K.; Yang, G.-P.; Wang, Y.-J. Adsorption of Dimethyl Phthalate on Marine Sediments. Water, Air, Soil Pollut. 2004, 157, $179-192$.

(2) Yang, F.; Wang, M.; Wang, Z. Sorption Behavior of 17 Phthalic Acid Esters on Three Soils: Effects of pH and Dissolved Organic Matter, Sorption Coefficient Measurement and QSPR Study. Chemosphere 2013, 93, 82-89.

(3) Liu, H.; Zhang, D.; Li, M.; Tong, L.; Feng, L. Competitive Adsorption and Transport of Phthalate Esters in the Clay Layer of JiangHan Plain, China. Chemosphere 2013, 92, 1542-1549.

(4) Banerjee, P.; Piwoni, M. D.; Ebeid, K. Sorption of Organic Contaminants to a Low Carbon Subsurface Core. Chemosphere 1985, 14, $1057-1067$.

(5) Li, B.; Qian, Y.; Bi, E.; Chen, H.; Schmidt, T. C. Sorption Behavior of Phthalic Acid Esters on Reference Soils Evaluated by Soil Column Chromatography. Clean: Soil, Air, Water 2010, 38, 425-429.

(6) Hunter, J. G.; Uchrin, C. G. Adsorption of Phthalate Esters on Soil at Near Saturation Conditions. J. Environ. Sci. Health, Part A: Toxic/Hazard. Subst. Environ. Eng. 2000, A35, 1503-1515.

(7) Maraqa, M. A. Effects of Fundamental Differences Between Batch and Miscible Displacement Techniques on Sorption Distribution Coefficient. Environ. Geol. 2001, 41, 219-228.

(8) Lammers, L. N.; Bourg, I. C.; Okumura, M.; Kolluri, K.; Sposito, G.; Machida, M. Molecular Dynamics Simulations of Cesium Adsorption on Illite Nanoparticles. J. Colloid Interface Sci. 2017, 490, 608-620.

(9) Cygan, R. T.; Liang, J.-J.; Kalinichev, A. G. Molecular Models of Hydroxide, Oxyhydroxide, and Clay Phases and the Development of a General Force Field. J. Phys. Chem. B 2004, 108, 1255-1266.

(10) Berendsen, H. J. C.; Grigera, J. R.; Straatsma, T. P. J. The Missing Term in Effective Pair Potentials. J. Phys. Chem. 1987, 91, 6269-6271.

(11) Åqvist, J. Ion-Water Interaction Potentials Derived from Free Energy Perturbation Simulations. J. Phys. Chem. 1990, 94, $8021-8024$.

(12) Smith, D. E., Dang, L. X. Computer simulations of $\mathrm{NaCl}$ association in polarizable water. J. Chem. Phys. 1994, 100, 3757-3766.

(13) Jorgensen, W. L.; Maxwell, D. S.; Tirado-Rives, J. Development and Testing of the OPLS All-Atom Force Field on Conformational Energetics and Properties of Organic Liquids. J. Am. Chem. Soc. 1996, 118, 11225-11236.

(14) Wu, Y.; Si, Y.; Zhou, D.; Gao, J. Adsorption of Diethyl Phthalate Ester to Clay Minerals. Chemosphere 2015, 119, $690-696$. 